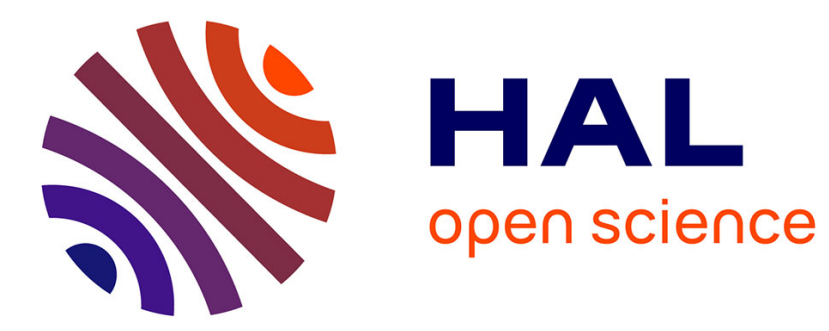

\title{
Nonlinear tensile behaviour of elementary hemp fibres. Part II : Modelling using an anisotropic viscoelastic constitutive law in a material rotating frame
}

Frédérique Trivaudey, Vincent Placet, Violaine Guicheret, Lamine Boubakar

\section{- To cite this version:}

Frédérique Trivaudey, Vincent Placet, Violaine Guicheret, Lamine Boubakar. Nonlinear tensile behaviour of elementary hemp fibres. Part II : Modelling using an anisotropic viscoelastic constitutive law in a material rotating frame. Composites Part A: Applied Science and Manufacturing, 2015, 68, pp.346 - 355. hal-02946382

\section{HAL Id: hal-02946382 \\ https://hal.science/hal-02946382}

Submitted on 23 Sep 2020

HAL is a multi-disciplinary open access archive for the deposit and dissemination of scientific research documents, whether they are published or not. The documents may come from teaching and research institutions in France or abroad, or from public or private research centers.
L'archive ouverte pluridisciplinaire HAL, est destinée au dépôt et à la diffusion de documents scientifiques de niveau recherche, publiés ou non, émanant des établissements d'enseignement et de recherche français ou étrangers, des laboratoires publics ou privés. 


\title{
Nonlinear tensile behaviour of elementary hemp fibres. Part II: Modelling using an anisotropic viscoelastic constitutive law in a material rotating frame
}

\author{
Frédérique Trivaudey, Vincent Placet*, Violaine Guicheret-Retel, M. Lamine Boubakar \\ FEMTO-ST Institute, Department of Applied Mechanics, UMR CNRS 6174, University of Franche-Comté, \\ F-25000 Besançon, \\ *Email: vincent.placet@univ-fcomte.fr \\ tel: +33 (0)3 81666055 fax: +33 (0)3 81666700
}

\begin{abstract}
A 3D viscoelastic model is presented, based on the use of finite element method for the study of the nonlinear tensile behaviour of hemp fibres. On the basis of an experimental investigation, Part I of this study (Placet, Cisse and Boubakar, Composites Part A 56:319-327, 2014) proposed a scenario based on several physical mechanisms, in order to explain the nonlinear behaviour of such fibres. These mechanisms included viscoelastic strain, cellulose microfibril reorientation, and shear strain-induced crystallisation of the amorphous paracrystalline components. The second part of this ongoing study proposes to implement such mechanisms and the associated constitutive laws in a simplified 3D model, in order to evaluate the contribution of each mechanism to the macroscopic tensile behaviour of the fibre.

The results show that the proposed anisotropic viscoelastic constitutive law, describing finite transformations through a material rotating frame formulation, is able to accurately simulate the shape of the experimentally observed tensile curves. This model is also used to investigate the influence of dislocation areas on the tensile behaviour and stress fields.
\end{abstract}

Keywords: Hemp fibres, B. Mechanical properties, C. Computational Modelling, C. Micro-mechanics 


\section{ABBREVIATIONS}

\author{
C: crystallization \\ D: default \\ E: elastic behaviour
}

\section{NOMENCLATURE}

$(\vec{X}, \vec{Y}, \vec{Z}):$ global coordinate system related to the fibre (Z: axial direction)

Mechanical tensors

$\underline{\sigma}$ : Cauchy true stress tensor

$\underline{\varepsilon}$ : total strain tensor

$\underline{\varepsilon}^{e}:$ elastic strain tensor

$\underline{\varepsilon}^{v e}:$ viscoelastic strain tensor

$\underline{\underline{S}}$ : elastic compliance tensor

$\underline{\underline{S}}^{v e}$ : viscoelastic compliance tensor

Elastic parameters

$\mathrm{E}_{\mathrm{L}}, \mathrm{E}_{\mathrm{T}}, v_{\mathrm{LT}}, v_{\mathrm{TT}}, \mathrm{G}_{\mathrm{LT}}$ : layer elastic properties (longitudinal and transverse moduli, Poisson's ratios, and shear modulus)

Viscoelastic parameters

$\beta_{\mathrm{T}}, \beta_{\mathrm{TT}}, \beta \mathrm{L}_{\mathrm{T}}$ : parameters characterizing the layer viscosity

$\xi_{i}(i \in \mathbb{N})$ : second order tensors corresponding to elementary mechanisms of viscoelastic flow

$\mu_{i}$ : weighing coefficients of the viscous mechanisms
MFA: cellulose microfibrils angle MFR: cellulose microfibrils reorientation VE: viscoelastic behavior

$\tau_{i}$ : release times of the viscous mechanisms $z n_{c}, 0, \Delta$ : shape parameters of triangular spectrum distribution of the relaxation times (centre, half-width, interval between two relaxation times)

$\sigma_{0}$ : constant axial stress.

$J_{Z Z_{\text {mod }}}(t)$ : computed axial viscoelastic compliance function

$J_{Z Z_{\text {exp }}}(t): \quad$ experimental axial viscoelastic compliance function

$J_{Z Z_{\text {mod }}}^{k}(k \in \mathbb{N}): \mathrm{k}^{\text {th }}$ computed value of axial viscoelastic compliance

$J_{Z Z_{\text {exp }}}^{k}: \mathrm{k}^{\text {th }}$ value of the experimental axial compliance data

\section{Transformation}

$\underline{\underline{F}}$ : deformation gradient

$\psi$ : supplementary angle of the microfibrils angle

$\vec{\chi}^{0}:$ microfibrils direction prior to transformation

$\vec{\chi}$ : microfibrils direction after transformation

$\underline{Q}_{f}:$ rotation tensor

$\left(\vec{e}_{01}, \vec{e}_{02}, \vec{e}_{03}\right)$ : Lagrangian frame

$\left(\vec{e}_{1}, \vec{e}_{2}, \vec{e}_{3}\right)$ : material frame

\section{INTRODUCTION}

Cellulose fibres from wood and plants represent a promising class of reinforcement materials, for use in high-performance polymer matrix composites. Because natural fibres are characterised by the strong variability of their mechanical properties and their high sensitivity to temperature and 
humidity, the design of reliable structure-based composites containing such natural fibres is a significant challenge for engineers, who are accustomed to using the consistent and accurate data available in the case of man-made fibres. These last ones are inherently produced in highlycontrolled conditions, unlike natural fibres.

In addition to their highly scattered mechanical properties, numerous fibres are characterised by nonlinear tensile behaviour. The understanding of this particular behaviour is of great importance, in view of the development of composites reinforced with plant fibres, since the behaviour of these fibres could have an impact on their performance when they are used to reinforce composite materials. Several authors have recently observed nonlinear behaviour in unidirectional composites reinforced with flax fibres (1-5). Some authors have also mentioned an increase in stiffness under cyclic loading, a behaviour which has been observed at the scale of individual fibres (6).

Many hypotheses have been proposed in the literature to explain the nonlinear tensile behaviour of isolated wood or plant fibres, and the fibres' stiffness recovery or improvement after loading beyond the yield point. These hypotheses were discussed in Part I of this study (7). In the case of wood tracheids, Page et al. (8) confirmed a relationship between the nonlinear shape of the tensile curve and the onset of cell wall buckling. They clearly showed that the yield point $\left(i_{1}\right)$ corresponds to the onset of wall buckling. More recently, Eder et al. (9) also showed that thick-walled fibres are highly resistant to tension buckling, and that this phenomenon can therefore explain the nonlinearity of the stress-strain curve only, in the case of a thin-walled fibre. Since hemp fibres generally have a thick wall, tension buckling therefore does not explain the nonlinearity of the stress-strain curve in the case of such fibres. Other authors have also attributed nonlinear behaviour to irreversible reorientations of the cellulose microfibrils with respect to the fibre axis in particular in the $S_{2}$ layer, when they are submitted to axial loading. $S_{2}$ is the main sub-layer of the cell wall in terms of its thickness and therefore contributes mainly to the global mechanical behaviour of the fibre. A linear fit was established between the microfibril angle (MFA) and strain for coir fibres, by Martinschitz 
et al. (10). Other authors have attributed this nonlinear behaviour to shear deformations in the noncrystalline region, which can partially damage the cell wall, or provoke a stick-slip phenomenon (11). This stick-slip mechanism proposed by Keckes et al. (11) operates like a 'molecular Velcro'. According to these authors, beyond the yield point $\left(i_{1}\right)$ the shear stress could provoke a viscous flow of the matrix. When the stress is released there would be no back-flow of the matrix, but a lock-in phenomenon associated with immediate bond re-formation in the fibrils' new position. This could explain irreversible strain without significant damage. This model supposes the presence of noncovalent interactions between hemicellulose chains attached to adjacent cellulose microfibrils. Altaner and Jarvis (12) proposed an alternative model in which hemicellulose chains bridging continuously from one microfibril aggregate to the next. However, the mechanism is different; the computed tensile curves are similar to those of the original model.

Nonlinear tensile behaviour was modelled by Nilsson and Gustafsson (13) for hemp fibres, by introducing defects into the helical structure of the cellulose microfibrils and assuming that the hemicellulose has an elasto-plastic constitutive relation. Navi and Sedighi-Gilani $(14,15)$ proposed a model for wood fibres with an elasto-plastic behaviour for amorphous polymers, based on the assumption of a helical, non-uniform distribution of cellulose microfibrils in the fibre and damage to the amorphous constituents after yielding. The origin of the dissipative behaviour of elementary fibres is thus attributed to plasticity by these authors.

In the Part I of this study, using experimental investigations we proposed a scenario centred on several physical and microstructural mechanisms, in order to explain the nonlinear behaviour (type 3) of such fibres, including viscoelastic strain, cellulose microfibril reorientation and shear straininduced crystallisation of the amorphous cellulose. In the present, second part of the study, we propose to implement such mechanisms, together with the associated constitutive laws, into a 3D model which can be used to evaluate the contribution of each physical mechanism to the macroscopic tensile behaviour of type 3 of the fibre. 


\section{MODELING}

\section{D model}

The macroscopic simplified 3D model used for this study is based on a previously developed model, described in detail in (16). The elementary hemp fibre is idealised as a single-layered, hollow, thick-walled cylinder made of an orthotropic material having a helical orientation (corresponding to the cellulose microfibril orientation). The possible variation in cross-section shape and size along the fibre length, non-unity transverse aspect ratios (i.e. non-circular crosssection) and their induced effects on the fibre mechanical behaviour (stress concentration, fibre rotation...) are therefore not addressed in the model. The transverse anisotropy introduced by nonunity aspect ratios is also in consequence ignored in this model. The wall is modelled as if it were a long fibre-reinforced composite material, made with a mixture of three different polymers. The wall properties are determined using a homogenisation technique (16). The values obtained for its elastic properties are summarized in Tab. 1. The fibres were considered to be free to rotate, and the influence of the boundary conditions on the computed value of the apparent modulus was studied in Part I of this paper. It was shown that although the testing device prevented the fibre from rotating during tensile tests, the experimentally determined value of the apparent E-modulus was more closely related to the numerical value determined using a free rotation. This was explained by the relatively strong hypothesis of cellulose microfibril continuity formulated when constructing the model.

Hygro-mechanical coupling was not considered in this version of the model.

The external diameter of the fibres is $13.3 \mu \mathrm{m}$, and that of the lumen is $4.46 \mu \mathrm{m}$. These nominal values were determined experimentally, and are consistent with the dimensions typically reported in the literature.

The model for the fibre wall was developed assuming conditions of anisotropic viscoelasticity, in order to correctly represent the fibre's behaviour, when it is observed under experimental 
conditions. Microstructural variations as a function of loading level, such as MFA and cellulose crystallinity, were also taken into account using the appropriate constitutive laws, which were then implemented in the finite element code Abaqus ${ }^{\circledR}$.

\section{Dislocation model}

It is well known that plant fibres have wall irregularities, which are commonly referred to as dislocations, nodes or kink bands. For hemp fibres, these dislocation areas are located mainly in the $\mathrm{S}_{2}$ layer (17) and represent from $12 \%$ to $21 \%$ of the total fibre wall (18). They are induced mainly by axial compression during the growth phase of the plant, and by the fibre separation process (19). Although the mechanisms responsible for these dislocations are not well understood, it is generally accepted that the cellulose microfibrils inside the dislocations are oriented differently than in the surrounding fibre wall. Using polarised Raman microspectroscopy, Thygesen and Gierlinger (20) have recently shown that the MFA ranges from approximately $5^{\circ}$ to $10^{\circ}$ in the bulk fibre wall, and is approximately $30^{\circ}$ in the central part of the dislocation. On each side of the dislocation, two regions were found with opposite winding directions and an MFA of approximately $10^{\circ}-15^{\circ}$.

On the basis of this observation, as well as the results from some of our measurements, an MFA of $11^{\circ}$ was used in our numerical simulations for the bulk regions of the fibre, and an MFA of $30^{\circ}$ was used for the dislocation areas.

As some of these simulations showed that the regions on either side of the dislocation (with opposite winding directions) have no significant influence on the fibre's tensile behaviour, these areas were not taken into account in the model presented here.

The dislocations were also considered to extend all the way across the fibre, and to make up $25 \%$ of the fibre wall. Dislocations are distributed along the fibre at approximately regular intervals. Again, since the numerical simulations showed that the fibre's behaviour is not significantly influenced by the distribution of these dislocations, these were modelled as if they were grouped together in the same area. 


\section{Constitutive laws}

\section{Visco-elasticity}

Viscoelastic model

Assuming that the local state of the material is completely defined by the elastic strain tensor $\underline{\varepsilon}^{e}$ and a set of second order tensors $\underline{\xi_{i}}(i \in \mathbb{N})$ corresponding to elementary mechanisms of viscoelastic flow, the state law leads to the following instantaneous constitutive equation :

$\underline{\dot{\varepsilon}}-\underline{\dot{\varepsilon}}^{v e}\left(\underline{\xi_{i}} ; \underline{\sigma}\right)=\underline{S} \dot{\underline{\sigma}}$,

where $\underline{\sigma}$ is the Cauchy true stress tensor, $\underline{\underline{S}}$ the elastic compliance tensor corresponding to a transverse isotropic behaviour with respect to the microfibrils direction, $\underline{\varepsilon}^{v e}$ the viscoelastic strain tensor and $\underline{\varepsilon}$ the total strain tensor such as $\underline{\varepsilon}=\underline{\varepsilon}^{e}+\underline{\varepsilon}^{v e}$.

Following (21), the viscoelastic flow rule is given by :

$\underline{\underline{\dot{\varepsilon}}}^{v e}=\sum_{i=1}^{n} \underline{\dot{\xi}}_{i}=\sum_{i=1}^{n} \frac{1}{\tau_{i}}\left(\mu_{i} \underline{\underline{S}}^{v e} \underline{\sigma}-\underline{\xi}_{i}\right)$

where $\underline{S}^{v e}$ is a viscoelastic compliance tensor whose general form is derived by considering an isotropic transverse behaviour with respect to the microfibrils direction, which is assumed to be purely elastic : 


$$
\underline{\underline{S}}^{v e}=\left(\begin{array}{cccccc}
0 & 0 & 0 & 0 & 0 & 0 \\
0 & \frac{\beta_{T}}{E_{T}} & -\frac{\beta_{T T} v_{T T}}{E_{T}} & 0 & 0 & 0 \\
0 & -\frac{\beta_{T T} v_{T T}}{E_{T}} & \frac{\beta_{T}}{E_{T}} & 0 & 0 & 0 \\
0 & 0 & 0 & \frac{\beta_{L T}}{G_{T}} & 0 & 0 \\
0 & 0 & 0 & 0 & \frac{\beta_{L T}}{G_{T}} & 0 \\
0 & 0 & 0 & 0 & 0 & \frac{\beta^{*}}{G_{T T}}
\end{array}\right) ; \frac{\beta^{*}}{G_{T T}}=2\left(\frac{\beta_{T}}{E_{T}}+\frac{\beta_{T T} v_{T T}}{E_{T}}\right)
$$

Such a tensor requires, in addition to the classical elastic parameters, the identification of three new parameters $\left(\beta_{T}, \beta_{T T}, \beta_{L T}\right)$ related to the viscoelastic behaviour.

Also in equation 2 , for a given i value, $\mu_{i}$ and $\tau_{i}$ represent respectively the relative weight and the corresponding release time.

A triangular spectral model is used in this work to define a distribution function of $\mu_{i}$ with respect to $\log \left(\tau_{i}\right)$ under the following condition:

$\sum_{i=1}^{n} \mu_{i}=1$

This kind of spectrum is defined by its centre $z n_{c}$ and its half-width $z n_{0}$ as shown in Fig. 1 where $\Delta$ depends on both these quantities and on the number $\mathrm{n}$ of elementary mechanisms. For the sake of accuracy, thirty-one elementary mechanisms are used.

\section{Parameter identification}

Two scalar independent creep functions have to be experimentally determined to characterize anisotropic time-dependent behaviour of hemp fibres. Laborious experimentations work has to be performed to determine these functions, in particular to determine the transverse creep function. In 
this study, we are only working with the experimentally determined axial creep function. The theoretical axial viscoelastic compliance is expressed as follows:

$$
J_{Z Z_{\mathrm{mod}}}(t)=\frac{\varepsilon_{Z Z}^{v e}(t)}{\sigma_{0}}=\frac{\sum_{i=1}^{31} \mu_{i} S_{Z Z}^{v e}\left(1-e^{-\frac{t}{\tau_{1}}}\right)}{\sigma_{0}} ; S_{Z Z}^{v e}=\frac{\beta_{T}}{E_{T}} \cos ^{4}(\psi)+\frac{\beta_{L T}}{G_{L T}} \cos ^{2}(\psi) \sin ^{2}(\psi)
$$

$\psi$ is the supplementary angle of the MFA $\left(90^{\circ}-M F A\right)$ and $\sigma_{0}$ the constant axial stress.

On the basis of the axial viscoelastic compliance, only two $\left(\beta_{T}, \beta_{L T}\right)$ of the three viscoelastic parameters can be identified (Eq. 5). These two viscoelastic parameters were identified from a creep test on a single hemp fibre, combined with the use of the aforementioned model and a hybrid minimisation algorithm. The results of a broad experimental campaign, made with elementary hemp fibres in order to accurately characterize their creep behaviour, will be presented in a forthcoming paper. For this study, a creep curve recorded on a single hemp fibre at a relative humidity of $50 \pm 5 \%$ and a temperature of $21 \pm 1{ }^{\circ} \mathrm{C}$ was used to identify the viscoelastic parameters. This test was made using a tensile machine (DMA Bose Electroforce 3230) with a clamping length of $10 \mathrm{~mm}$ and a constant load of $30 \mathrm{mN}$. The axial strain was determined as the fibre elongation divided by its initial length, and was recorded as a function of time for a period of 3000 seconds.

In order to avoid convergence towards local optima in the case of multimodal test functions, the Levenberg-Marquardt algorithm was combined with a heuristic method based on a genetic algorithm. This hybrid algorithm is described in $(22,23)$, and involves minimisation of the objective function $\mathrm{f}$ (Eq. 6).

$f=\frac{\sqrt{\sum_{k=1}^{n}\left(\frac{J_{Z Z_{\text {exp }}}^{k}-J_{Z Z_{\text {mod }}}^{k}}{\max \left(J_{Z Z_{\text {exp }}}^{k}\right)}\right)^{2}}}{2 n}$ 
where $J_{Z Z_{\text {exp }}}^{k}$ is the $\mathrm{k}^{\text {th }}$ value of the experimental compliance data, $J_{Z Z_{\bmod }}^{k}$ is the $\mathrm{k}^{\text {th }}$ computed value using the aforementioned model, and $n$ is the number of elements in the dataset.

Fig. 2 shows that by combining this spectral model with a suitable identification technique, a relatively good fit can be achieved with the experimental data. A broad study on viscoelastic spectral models will soon be submitted for publication. It will show that a better fit can be achieved with the experimental data when using Gaussian or parabolic spectra. For this study, the timedelayed response identified with the simple triangular spectral model is sufficient to ensure a suitable representation of the experimental behaviour. The identified parameters are summarized in Tab. 1.

\section{Evolution law of MFA}

Although the relationship between the initial MFA and the initial or tangent apparent modulus of fibres placed under tensile loading has been widely investigated, both experimentally and theoretically, very little research has been devoted to the study of MFA variations in the same fibre during tensile tests. From the experimental point of view, this type of study is particularly difficult to perform and requires the use of synchrotron radiation when an X-ray scattering technique is used to measure the MFA. Contradictory results have been published in the literature. Contrary to the results of Kölln et al. (24), Astley and Donald (25) did not observe a change in the distribution of flax fibre orientations when they were placed under strain. In a previous study using WAXS, we measured a slight decrease in MFA (approximately $3^{\circ}$, from $10.8^{\circ}$ to $7.8^{\circ}$, for an axial strain varying from $0 \%$ to $2 \%$ ) with a bundle of hemp fibres, mainly in the first portion of the tensile curve (26). These results, recorded at the scale of a fibre bundle, cannot be generalized to the case of a single fibre. In the first part of this study, using in situ polarised light microscopy (PLM) on single hemp fibres, it was also observed that some of the microfibrils became realigned. This type 
of quantitative approach should be interpreted with considerable caution, since PLM allows mainly the microfibrils located on the fibre wall surfaces to be observed, and not those of the $\mathrm{S}_{2}$ layer.

As a consequence of the difficulties encountered in determining a law to provide an accurate description of the variations observed in the MFA, in the present study we propose a consistent modelling based on kinematic and material considerations. To the best of the authors' knowledge, this is the first time a theoretical model has been proposed to describe this phenomenon.

The proposed approach assumes that the fibre is locally subjected to finite transformations which involve large rotations and small elastic-viscoelastic strains. In the context of an incremental computation, the previous constitutive framework (Eqs. 1, 2, 3) remains valid in a material rotating frame whose one of the axis coincides with the microfibrils direction.

In this study, the evolution law of the cellulose microfibrils is derived from the intrinsic expression of the deformation gradient $\underline{\underline{\boldsymbol{F}}}$ in the case of a plane kinematics with respect to the radial direction. Hence, by using a lagrangian frame $\left(\overrightarrow{\boldsymbol{e}}_{\mathbf{0 1}}, \overrightarrow{\boldsymbol{e}}_{\mathbf{0 2}}, \overrightarrow{\boldsymbol{e}}_{\mathbf{0 3}}\right)$ and a material one $\left(\overrightarrow{\boldsymbol{e}}_{\mathbf{1}}, \overrightarrow{\boldsymbol{e}}_{\mathbf{2}}, \overrightarrow{\boldsymbol{e}}_{\mathbf{3}}\right)$ such as $\overrightarrow{\boldsymbol{e}}_{\mathbf{0 1}}$ and $\overrightarrow{\boldsymbol{e}}_{\mathbf{1}}$ define the same material line during the transformation and $\overrightarrow{\boldsymbol{e}}_{\mathbf{0 2}}$ (respectively $\overrightarrow{\boldsymbol{e}}_{\mathbf{2}}$ ) remains in the plane perpendicular to the radial direction $\overrightarrow{\boldsymbol{e}}_{\mathbf{0 3}}=\overrightarrow{\boldsymbol{e}}_{\mathbf{3}}$ (see Fig. 3), the deformation gradient takes the following form :

$$
\underline{F}=\left(\begin{array}{ccc}
F_{11} & F_{12} & 0 \\
0 & F_{22} & 0 \\
0 & 0 & F_{33}
\end{array}\right)_{\vec{e}_{i}, \vec{e}_{i}}
$$

Let $\vec{\chi}^{0}$ be the microfibrils direction prior to transformation, and let the two directions $\vec{e}_{1}^{0}$ and $\vec{\chi}^{0}$ be separated by an initial reference angle $\psi^{0}=90^{\circ}-M F A$. When the rotation $Q_{f}$ is considered, the new microfibrils direction becomes:

$\vec{\chi}=\underline{Q}_{f} \vec{\chi}^{0}$ 
and the direction $\vec{a}_{1}=\underline{Q}_{f} \vec{e}_{1}^{0}$ are still separated by the angle $\psi_{0}$.

Hence :

$\underline{Q_{f}}=\vec{\chi}_{i} \otimes \vec{\chi}_{i}^{0}=Q_{i j} \vec{e}_{i} \otimes \vec{e}_{j}^{0}$

where $Q_{11}=\cos \left(\psi-\psi^{0}\right), Q_{12}=-\sin \left(\psi-\psi^{0}\right), Q_{21}=\sin \left(\psi-\psi^{0}\right)$ and $Q_{22}=\cos \left(\psi-\psi^{0}\right)$.

Since the microfibrils direction is a material one, the direction $\vec{\chi}$ can also be computed by the following expression :

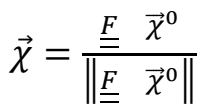

From Eq.8 and Eq. 10, it comes finally:

$$
\tan \psi=\frac{F_{22} \sin \psi^{0}}{F_{11} \cos \psi^{0}+F_{12} \sin \psi^{0}}
$$

\section{Strain-induced crystallisation law}

In addition to the change in MFA under tensile loading, we also take into account any possible crystallisation of the paracrystalline polymers. Indeed, using in situ X-ray scattering on dry flax fibres, Astley and Donald (25) have shown that strain-induced crystallization of the cellulose microfibrils can occur when the fibre is subjected to tensile strain. The synchrotron measurements performed by these authors provide evidence that non-crystalline cellulose chains are initially oriented. The existence of non-cellulosic, highly ordered paracrystalline domains has also been revealed by proton spin relaxation time calculations, made by Duchemin et al. (27). Using an X-ray diffraction technique, Toba et al. (28) also showed that cyclic sorption can promote the crystallization of cellulose microfibrils in wood cell walls, regardless of heating. In fact, it is well known that heat treatment can lead to the degradation of the amorphous cellulose surrounding the 
cellulose microfibrils, thus inducing a change in their crystallinity. These authors concluded that non-crystalline cellulose progressively forms hydrogen bonds with the cellulose at the surface of the crystalline region during sorption cycling, thus increasing the size of the crystal.

To the best of the present authors' knowledge, there is currently no evidence in the literature of strain-induced crystallization in hemp fibres, when these are subjected to tensile loading. However, when measurements performed on flax fibres and wooden materials are considered, the hypothesis proposed in Part I of this study, according to which crystallization could occur under mechanical or hygro-thermal loading of the highly ordered paracyrstalline components, appears to be relevant.

In view of the lack of existing experimental data (both quantitative and phenomenological), it is proposed, in the present study, to consider an arbitrary linear variation of the crystallinity ratio, as a function of shear strain. It is assumed that this ratio increases linearly from $65 \%$ to $85 \%$, when the in-plane strain shear increases from $0.5 \%$ to $4 \%$.

\section{FE Modelling}

Several 3D (8- or 20-node) meshes were tested using the FE Abaqus ${ }^{\circledR}$ Code to represent the fibre, using the model described above (see (16) for the corresponding numerical modelling). When the elastic axial, hoop and shearing stresses aligned in the radial direction of the fibre are compared with the analytical elastic solution (16), and the modelling and computation time requirements are compared, a one-8nodes-element mesh in the direction of the fibre radius appears to provide a good compromise. In order to follow the fibre's curved geometry, 40 elements are used to represent its circumference. A total of 80 elements are used in the axial direction, to accurately model any defects present along the fibre's length. Fig. 3 provides an example of an undeformed, and a deformed mesh. The micro-fibril direction is defined with respect to this direction $\left(\psi^{0}=79^{\circ}\right)$, rather than the axial direction $\left(\mathrm{MFA}=11^{\circ}\right)$ used for the FE calculations. The calculations are made 
using either an elastic or a visco-elastic behaviour, with or without MFA updating, and with or without the assumption of the crystallinity ratio increasing as a function of fibre deformation. Any experimentally observed defects along the length of the fibre are modelled by an area in which the mechanical behaviour is the same as in the bulk area of the fibre, but the MFA is smaller than in the fibre $\left(\psi^{0}=60^{\circ}\right)$. The initial angle in the zone having a defect was tested for different MFA values, over the range $\left(50^{\circ}-70^{\circ}\right)$, with a corresponding percentage of defects lying in the range between $25 \%$ and $75 \%$.

The loading is applied by imposing displacements at the both ends of the sample; a global axial strain is obtained by computing the ratio of the displacement to the length of the sample. The strain rate is of the same order of magnitude as the experimentally observed value, i.e. approximately $5.10^{-4} \mathrm{~s}^{-1}$

In order to compare these calculations with our experimental results, the global axial stress was computed by summing the reactions - to the imposed displacement - at all nodes belonging to the end surface. This force was then divided by the surface area of the sample, to obtain the axial stress. For each load increment, the tangent of the apparent Young's modulus was obtained by computing the ratio between the stress increment and the strain increment. In order to correlate the simulations with the experimentally determined values, the fibre's mechanical properties were determined using its initial values of length and radius.

\section{RESULTS AND DISCUSSION}

FE simulations were carried out using different constitutive laws, using the elastic and viscoelastic parameters listed in Tab. 1. The results are summarised in Figs. 4 and 5, where Figs. 4.a and 5.a. show the computed tensile stress variations as a function of tensile strain, and Figs. 4.b. and 5.b show the change in apparent tangent Young's modulus as a function of tensile strain. Two experimental curves selected among a database collected during an extensive experimental 
campaign on elementary hemp fibres, are also plotted in these figures. They reveal the strong discrepancies observed during the experimental campaign. Using the described model, we propose to assess the degree to which each physical mechanism governs the tensile response of the fibre.

\section{$\underline{\text { Elastic behaviour }(\mathbf{E})}$}

When elastic behaviour is considered for the homogenized materials comprising the fibre wall, a quasi-linear curve is observed in the stress-strain plot. Fig. 4.b. shows that the apparent rigidity increased slightly when the strain was increased, from an initial modulus of approximately $40 \mathrm{GPa}$ to a tangent modulus of $47 \mathrm{GPa}$ at $4 \%$ axial strain. This stiffening is due to the structural effect resulting from the fibre's cylindrical geometry, the helical organization of the cellulose microfibrils, and the change in cross-sectional area as a function of tensile loading. The structural effect was extensively analysed in a previous study (16). It can be seen that the simulated behaviour is quite different to that observed experimentally.

\section{Cellulose Microfibril Reorientation (MFR)}

The variations in MFA as a function of axial strain were determined using the proposed theoretical model. During the tensile test, the microfibrils rotated from their initial angle of $11^{\circ}$ to an angle of $5.7^{\circ}$, when placed under the greatest value (4\%) of axial strain (Fig. 6). These computed values are in very good agreement with those measured on hemp bundles using the WAXS technique. Using the WAXS technique on bundle of hemp fibres, a decrease in MFA, from $10.8^{\circ}$ to $7.8^{\circ}$, was measured when the axial strain varied from 0 to $2 \%$. The computed values of strain, ranging from $11^{\circ}$ to $8^{\circ}$, thus lie in the same range.

Fig. 4.a. clearly shows that when this progressive decrease in MFA is taken into account during the tensile test, the resulting curve has a slightly parabolic shape, which is similar to that obtained in part III of the experimental tensile curve. The final value of the apparent modulus (64 GPa), 
corresponding to an axial strain of $4 \%$, is approximately 1.6 times higher than the initial value (40 GPa), see Fig. 4.b. This degree of stiffening is consistent with the experimental results obtained by the present authors with Repeated Progressive Loading $(29,30)$, and is of the same order as that observed by Baley (6) and Silva (31), with flax and sisal fibres, respectively.

The simulated behaviour is nevertheless quite different to that observed experimentally.

\section{Viscoelasticity}

\section{Viscoelasticity in the case of small strain hypothesis (VE)}

The viscoelastic law was identified during an earlier experimental campaign based on a creep test with an elementary hemp fibre. The identified parameters are summarized in Tab. 1. On the basis of the axial viscoelastic compliance, only two $\left(\beta_{T}, \beta_{L T}\right)$ of the three viscoelastic parameters were identified. A sensitivity analysis on the $\beta_{\text {Tт }}$ parameter was performed. Our numerical simulations showed that the axial viscoelastic compliance is not sensitive to this parameter $\beta_{\mathrm{TT}}$ when its value is comprised between 1 and 25. An additional work based on a parametric identification with the 3D FE model is underway to identify the three viscoelastic parameters from experimental creep function. The results will be published in an upcoming article.

Using the viscoelastic law, significant nonlinearity was revealed in the stress-strain plot (Fig. 4.a., VE, red curve).

The apparent modulus decreased from $40 \mathrm{GPa}$ to approximately $20 \mathrm{GPa}$ during the first $2 \%$ of strain, and then had a slight tendency to increase beyond $2 \%$ of strain. Although the first part of the curve is in relatively good agreement with the upper experimental curve, the stiffening observed after the inflection point is not sufficient to ensure a well-adjusted fit with the experimental results. This result is nevertheless interesting, since the viscoelastic behaviour can to a certain extent explain the nonlinear tensile behaviour observed in wood or plant fibres, in particular the stiffening which occurs following an initial decrease in rigidity. 


\section{Viscoelasticity in the case of finite transformations (VE/MFR)}

When the viscoelastic behaviour of the homogenised material of the fibre wall is combined with the MF re-alignment phenomenon associated with tensile loading, a strongly nonlinear curve is obtained in the stress-strain plot (Fig. 5.a., VE/MFR). The shape of the simulated tensile curve (VE/MFR) is very close to that observed experimentally (Type 3). The first part of the curve is linear until the first yield level, beyond which a strong decrease in rigidity is observed. A second inflection point (corresponding to the experimentally observed point $\mathrm{i}_{2}$ ) appears at a higher strain level, and is followed by a parabolic increase in rigidity up until final failure. This result clearly shows that the nonlinear tensile behaviour of plant or wood fibres can be described and simulated by a viscoelastic law for finite transformations, and that the main source of dissipative behaviour is certainly viscoelasticity, rather than plasticity as proposed by some authors $(13,14)$. This result is in good agreement with our experimental observations, the time-dependent mechanisms in particular, and tends to validate the scenario proposed in the first part of this study.

In a previous study, we also observed that the proportion of curves of type 3 increases significantly as a function of the fibre's water content (32). It was assumed that, by plasticizing amorphous components of the cell wall and thereby activating their viscoelasticity, water could facilitate the realignment of cellulose microfibrils. Our numerical simulations clearly reveal that MF realignment is increased by the viscosity ratio. MF re-orientations occur more rapidly and have higher angular values for a given value of axial strain, when the material is assumed to be viscoelastic. As shown in Fig. 6, the MFA decreased from an initial value of $11^{\circ}$ to $4.2^{\circ}$ in the case of an axial strain of $3 \%$, whereas it reached a value of only $6.7^{\circ}$ in the case of purely elastic behaviour.

It should be noted that the parabolic increase in rigidity which can be observed at high values of strain on the tensile curve (Fig. 5a) is lower than that observed for the upper experimental curve. In 
this figure, it can be seen that a good fit is obtained (VE/MFR/C, light green curve) when the potential shear-induced crystallisation is taken into account.

Fig. 7 shows the stress-strain and apparent tangent modulus - strain curves for different strain rates from $5.10^{-5}$ to $5.10^{-3} \mathrm{~s}^{-1}$. As expected and as for all polymeric materials, the fibre response depends directly on the strain rate. Our results show that the degree of nonlinearity decreases with an increase in strain rate.

\section{Influence of dislocation areas on the tensile behaviour}

The influence of dislocation areas on the tensile behaviour of hemp fibres has not been fully characterised. In living plants, it is speculated that these have no detrimental effect, and could perhaps be beneficial (20). In the case of isolated fibres, no clear tendency has been demonstrated in the literature. At present, a small number of published results suggest that the fibre's strength is not affected by an increase in the proportion of dislocation areas (33). However, experimental investigations are particularly difficult to implement and interpret, due to the widely scattered results. In the present study, it is proposed to analyse the influence of dislocations on the mechanical behaviour of single fibres, using the proposed model. The results of these numerical simulations shed new light on this topic.

If a dislocation proportion of $25 \%$ is assumed, in association with an MFA of $30^{\circ}$, a strong decrease in apparent tangent modulus is observed (VE/MFR/C/D Fig. 5.a.). The initial modulus is approximately $27 \mathrm{GPa}$, and decreases to $14 \mathrm{GPa}$ at $1 \%$ axial strain, following which it increases gradually up to $25.5 \mathrm{GPa}$ at $3 \%$ axial strain. The shape of this simulated tensile curve is well adjusted to the lower experimental curve. In view of these numerical results, when they are compared with the behaviour of bulk fibre having a constant and relatively small MFA along its length, it can be clearly understood that the presence of dislocations leads to a significant decrease in the apparent stiffness of the fibres. It can thus be concluded that in the case of hemp fibres, an increase in the proportion of dislocations contributes to a decrease in the fibre's apparent rigidity. 
Fig. 8 also shows that the amplitude of the reorientations is almost twice as strong in the dislocation area as in the bulk area. The decrease in MFA, corresponding to an axial strain ranging from 0 to $4 \%$, is respectively $14^{\circ}$ and $7.5^{\circ}$ in these two areas. The difference in MFA in these two areas, for the higher strain values, is thus significantly reduced. This result is in agreement with the in situ PLM observation described in Part I of this study, which showed that the dislocations gradually disappear during tensile testing, as a result of MFA homogenization along the length of the fibre. When a purely elastic behaviour is considered, a significant stress concentration (Figs. 9.a. and 10.a.), together with an increase in the axial stress gradient within the thickness of the cell wall (Fig. 9.b.), are observed in the dislocation area. It thus seems clear that these areas could be preferential failure initiation zones. Conversely, when viscoelastic behaviour is assumed for large transformations, the axial stress level is found to be relatively constant along the length of the fibre (Fig. 9.a. and Fig. 10.b.), and the axial stress gradient is nearly the same in both areas. Although this constitutive law accurately reflects the underlying physical mechanism, it is clear that, contrary to all expectations, dislocation areas do not induce significant stress concentrations. This numerical result could provide an explanation for the absence of any statistically significant influence of dislocations on the experimentally observed behaviour of the fibre's strength (33). 


\section{CONCLUSIONS}

The results presented in this paper show that a 3D hollow model with a fibre-reinforced viscoelastic constitutive law for large transformations is able to simulate the nonlinear tensile behaviour of single hemp fibres. Very good agreement in the curve shape is found with the experimental results. The model is able to successfully bound the batch of experimental curves. As hypothesised in Part I of this study, based on experimental investigations, the numerical results confirm the dominant role of viscoelastic phenomena in cellulose MF re-orientations, and the associated degree of nonlinearity.

This study also highlights the influence of dislocation areas on the mechanical behaviour of single fibres, in particular on their apparent rigidity. Both initial and tangent apparent rigidity up to failure are influenced by the presence of dislocations. The results also show that when elastic behaviour is assumed, the induced stress concentration and gradient in the dislocation area, associated with viscoelastic behaviour, are cancelled. This could explain why, contrary to all expectations, dislocation areas are not preferential areas of failure initiation.

The next parts of this paper series on the tensile behaviour of plant fibre will be focused on the modelling of other tensile responses, such as Type 2, and also to the modelling of tensile unloading with irreversible residual strains. The implementation of damage laws and visco-plastic mechanisms constitutes an interesting track to explore this subject. It could also be interesting to evaluate the influence of morphological aspects (such as non-unity transverse aspect ratios, discontinuities in the cross-section shape and size along the fibre length and so on) on the tensile response, the ultimate goal being to use the real 3D morphology of a single isolated fibre. 


\section{REFERENCES}

1. Liang S, Gning PB, Guillaumat L. A comparative study of fatigue behaviour of flax/epoxy and glass/epoxy composites. Composites Science and Technology 2012; 72 (5): 535.

2. Shah DU, Schubel PJ, Clifford MJ. Can flax replace E-glass in structural composites? A small wind turbine blade case study. Composites Part B: Engineering 2013; 52 (0): 172.

3. Shah DU, Schubel PJ, Licence P, Clifford MJ. Determining the minimum, critical and maximum fibre content for twisted yarn reinforced plant fibre composites. Composites Science and Technology 2012; 72 (15): 1909.

4. Shah DU, Schubel PJ, Clifford MJ, Licence P. The tensile behavior of off-axis loaded plant fiber composites: An insight on the nonlinear stress-strain response. Polymer Composites 2012; 33 (9): 1494.

5. Baets J, Plastria D, Ivens J, Verpoest I. Determination of the optimal flax fibre preparation for use in UD flax-epoxy composites. ECOCOMP, 4th International Conference on Sustainable Materials, Polymers and Composites. Birmingham, United Kingdom., 2011: 1.

6. Baley C. Analysis of the flax fibres tensile behaviour and analysis of the tensile stiffness increase. Composites Part A: Applied Science and Manufacturing 2002; 33 (7): 939.

7. Placet V, Cisse O, Boubakar L. Nonlinear tensile behaviour of elementary hemp fibres. Part I: Investigation of the possible origins using repeated progressive loading with in situ microscopic observations. Composites Part A: Applied Science and Manufacturing 2014; 56: 319.

8. Page DH, EL-Hosseiny F, Winkler K. Behaviour of Single Wood Fibres under Axial Tensile Strain. 1971; 229 (5282): 252.

9. Eder M, Stanzl-Tschegg S, Burgert I. The fracture behaviour of single wood fibres is governed by geometrical constraints: in situ ESEM studies on three fibre types. Wood Science and Technology 2008; 42 (8): 679.

10. Martinschitz K, Boesecke P, Garvey C, Gindl W, Keckes J. Changes in microfibril angle in cyclically deformed dry coir fibers studied by in-situ synchrotron X-ray diffraction. Journal of Materials Science 2008; 43 (1): 350.

11. Keckes J, Burgert I, Fruhmann K, et al. Cell-wall recovery after irreversible deformation of wood. 2003; 2 (12): 810.

12. Altaner CM, Jarvis MC. Modelling polymer interactions of the 'molecular Velcro' type in wood under mechanical stress. Journal of Theoretical Biology 2008; 253 (3): 434.

13. Nilsson T, Gustafsson PJ. Influence of dislocations and plasticity on the tensile behaviour of flax and hemp fibres. Composites Part A: Applied Science and Manufacturing 2007; 38 (7): 1722.

14. Sedighi-Gilani M. A micromechanical approach to the behavior of single wood fibers and wood fracture at cellular level. Lausanne: EPFL, 2006.

15. Sedighi-Gilani M, Navi P. Experimental observations and micromechanical modeling of successivedamaging phenomenon in wood cells' tensile behavior. Wood Science and Technology 2007; 41 (1): 69.

16. Placet V, Trivaudey F, Cisse O, Gucheret-Retel V, Boubakar ML. Diameter dependence of the apparent tensile modulus of hemp fibres: A morphological, structural or ultrastructural effect? Composites Part A: Applied Science and Manufacturing 2012; 43 (2): 275.

17. Thygesen LG, Bilde-Sörensen JB, Hoffmeyer P. Visualisation of dislocations in hemp fibres: A comparison between scanning electron microscopy (SEM) and polarized light microscopy (PLM). Industrial Crops and Products 2006; 24 (2): 181.

18. Thygesen L, Asgharipour M. The effects of growth and storage conditions on dislocations in hemp fibres. Journal of Materials Science 2008; 43 (10): 3670.

19. Thygesen L. Quantification of dislocations in hemp fibers using acid hydrolysis and fiber segment length distributions. Journal of Materials Science 2008; 43 (4): 1311.

20. Thygesen LG, Gierlinger N. The molecular structure within dislocations in Cannabis sativa fibres studied by polarised Raman microspectroscopy. Journal of Structural Biology 2013; 182 (3): 219.

21. Boubakar ML, Vang L, Trivaudey F, Perreux D. A meso-macro finite element modelling of laminate structures: Part II: time-dependent behaviour. Composite Structures 2003; 60 (3): 275.

22. Carbillet S. Contribution aux calculs fiabilistes sur des structures composites.: Franche-Comté, 2005. 
23. Richard F. Identification du comportement et évaluation de la fiabilité des composites stratifiés.: Franche-Comté, 1999.

24. Kölln K, Grotkopp I, Burghammer M, et al. Mechanical properties of cellulose fibres and wood. Orientational aspects in situ investigated with synchrotron radiation. Journal of Synchrotron Rdiation 2005; 12: 739.

25. Astley OM, Donald AM. The tensile deformation of flax fibres as studied by X-ray scattering. Journal of Materials Science 2003; 38 (1): 165.

26. Placet V, Bouali A, Garcin C, Cote J-M, Perré• P. Suivi par DRX des réarrangements microstructuraux induits par sollicitations mécaniques dans les fibres végétales tirées du chanvre. 20ème Congrès Français de Mécanique. Besançon, France, 2011.

27. Duchemin Bt, Thuault A, Vicente Al, Rigaud B, Fernandez C, Eve S. Ultrastructure of cellulose crystallites in flax textile fibres. Cellulose 2012; 19 (6): 1837.

28. Toba K, Yamamoto H, Yoshida M. Crystallization of cellulose microfibrils in wood cell wall by repeated dry-and-wet treatment, using X-ray diffraction technique. Cellulose 2013; 20 (2): 633.

29. Placet V. Characterization of the thermo-mechanical behaviour of Hemp fibres intended for the manufacturing of high performance composites. Composites Part A: Applied Science and Manufacturing 2009; 40 (8): 1111.

30. Placet V. Tensile behaviour of natural fibres. Effect of loading rate, temperature and humidity on the "accommodation" phenomena. International Conference on Experimental Mechanics. Poitiers, 2010.

31. Silva FdA, Chawla N, de Toledo Filho RD. An experimental investigation of the fatigue behavior of sisal fibers. Materials Science and Engineering: A 2009; 516: 90.

32. Placet V, Cisse O, Boubakar ML. Influence of environmental relative humidity on the tensile and rotational behaviour of hemp fibres. Journal of Materials Science 2012; 47 (7): 3435.

33. Thygesen L, Eder M, Burgert I. Dislocations in single hemp fibres - investigations into the relationship of structural distortions and tensile properties at the cell wall level. Journal of Materials Science 2007; 42 (2): 558. 
Figure 1: Spectral model for the release times

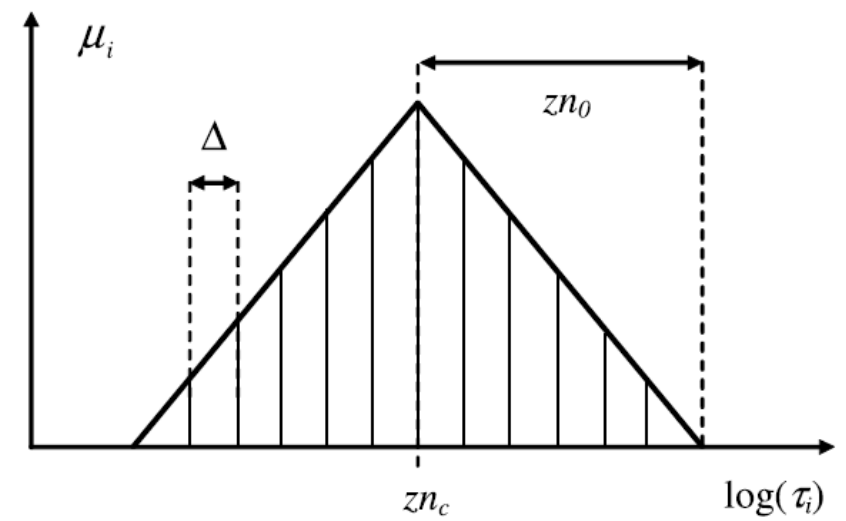


Figure 2: Identification of the viscoelastic parameters - comparison between the experimental and fitted curves.

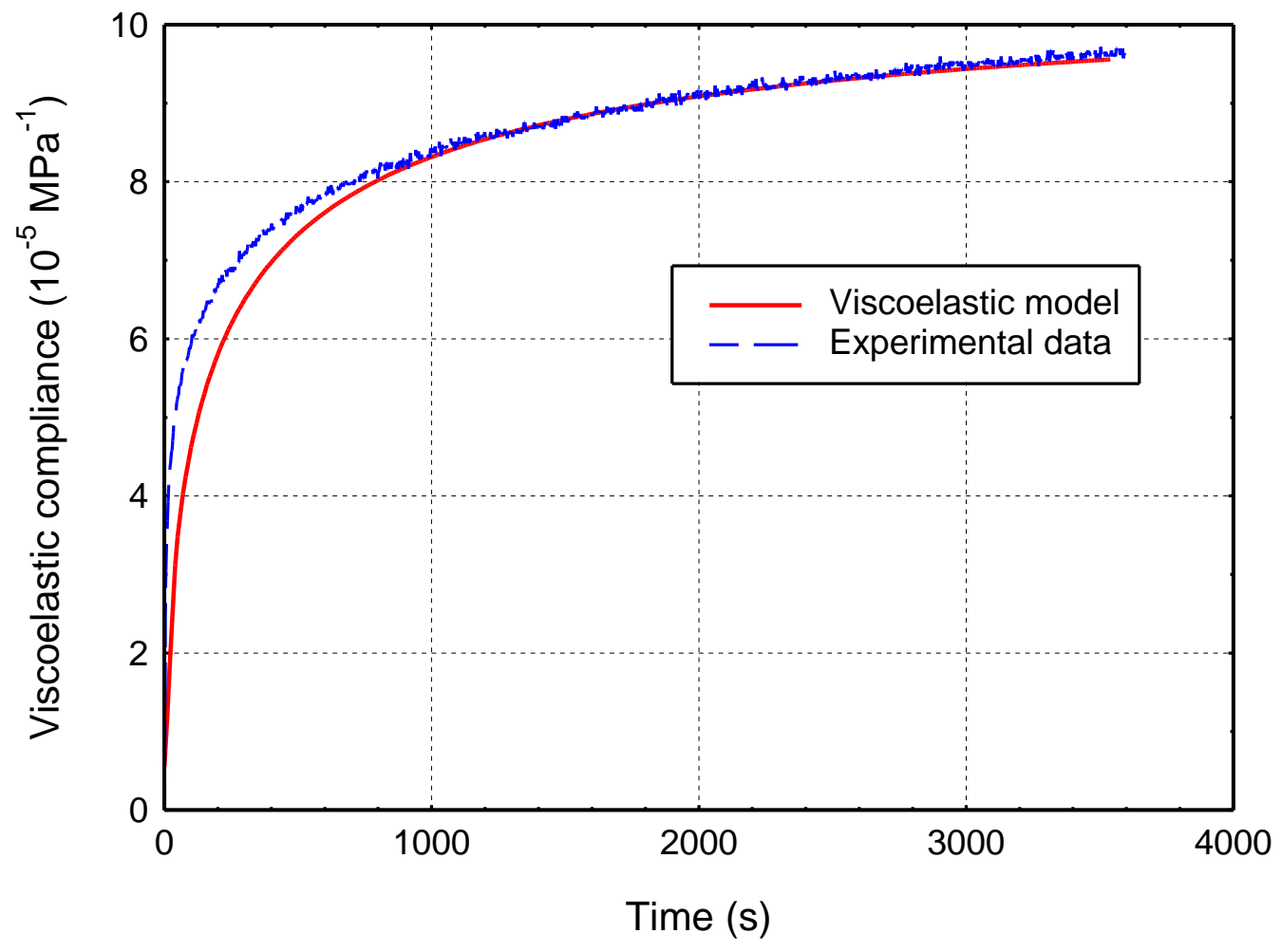


Figure 3: Theoretical variation of the microfibril's orientation after loading.

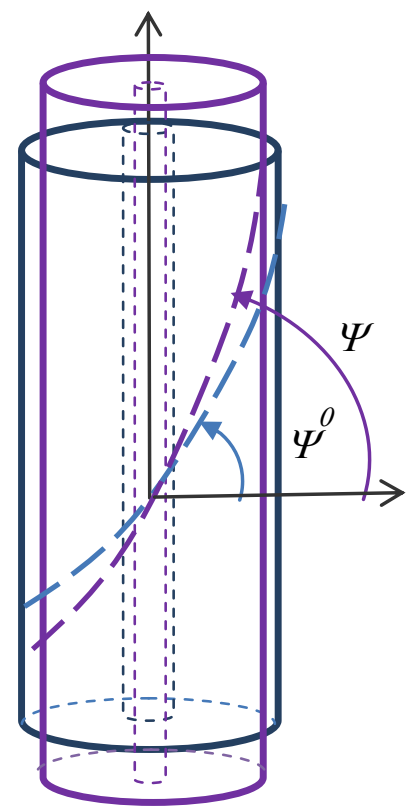

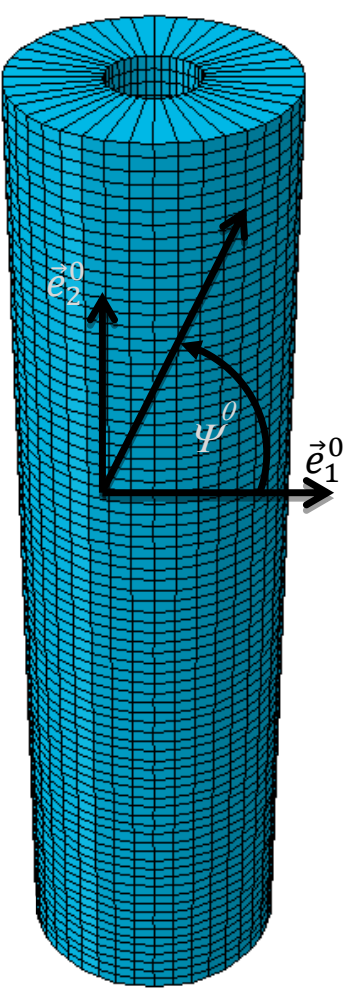

Undeformed sample

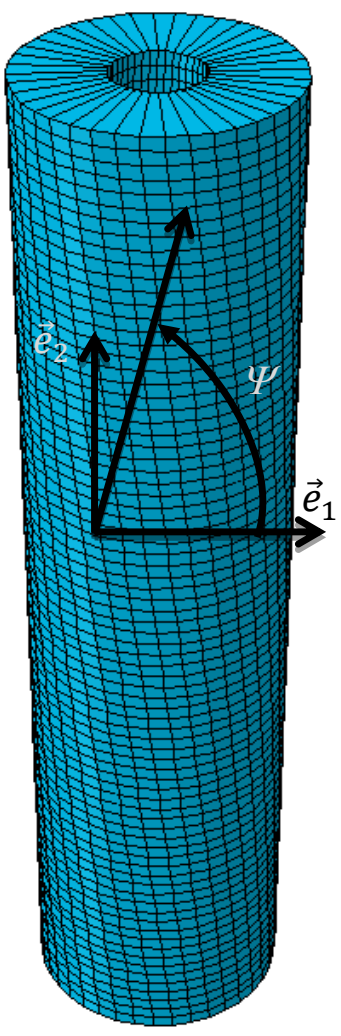

Deformed sample

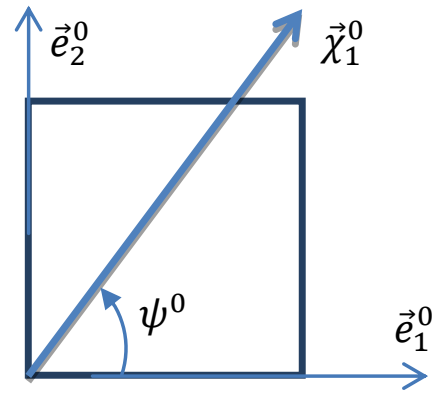

Before loading

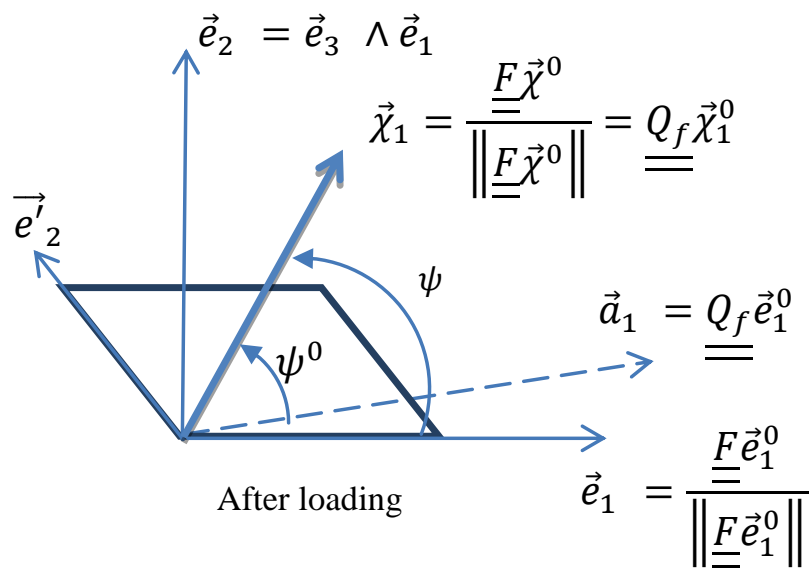


Figure 4. a. Stress-strain curves. b. Apparent modulus - strain curves. The curves are computed for different constitutive laws: E (Elastic), VE (Viscoelastic), MFR (microfibril reorientation). Two experimental curves selected among our database are also shown.
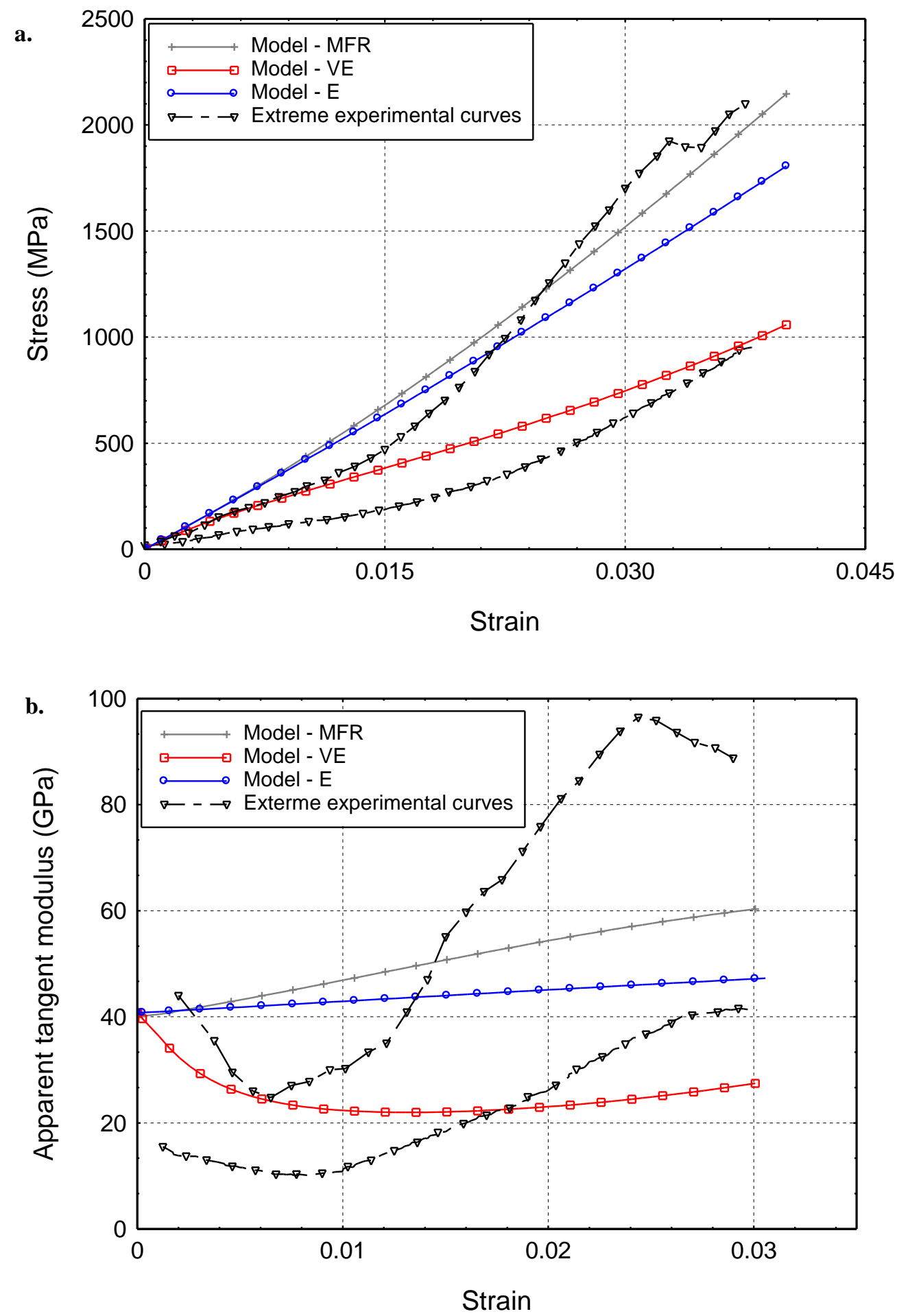
Figure 5. a. Stress-strain curves. b. Apparent modulus - strain curves. Computed curves for different constitutive laws: VE/MFR (Viscoelastic and Microfibril reorientation), VE/MFR/C (Viscoelastic and microfibril reorientation and stress-induced crystallisation), VE/MFR/C/D (Viscoelastic and Microfibrils Reorientation and stress-induced cystallisation with a dislocation area). The two experimental curves selected among our database are also shown.
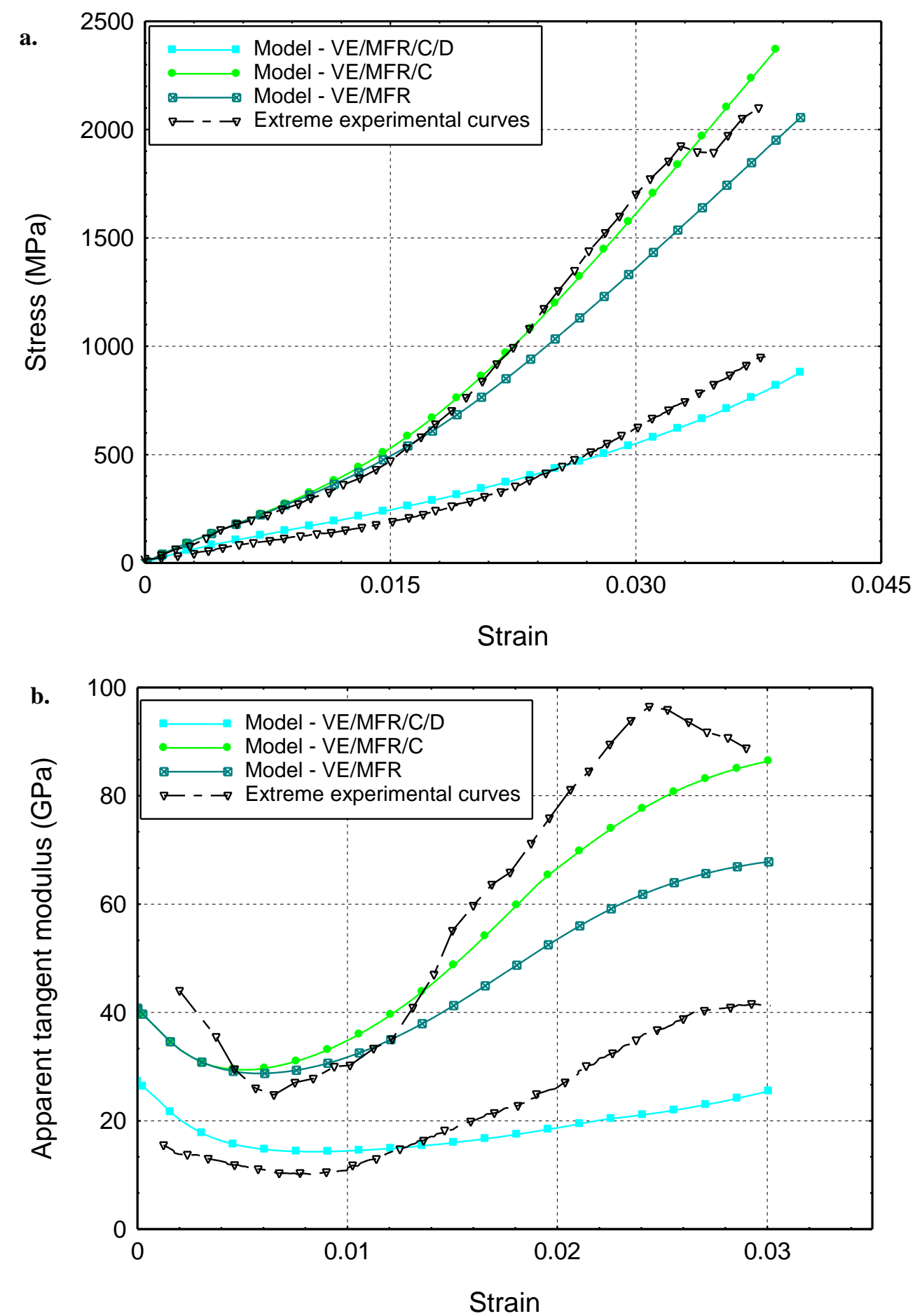
Figure 6: Computed MFA as a function of axial strain for two constitutive laws: elastic (E) and viscoelastic (VE).

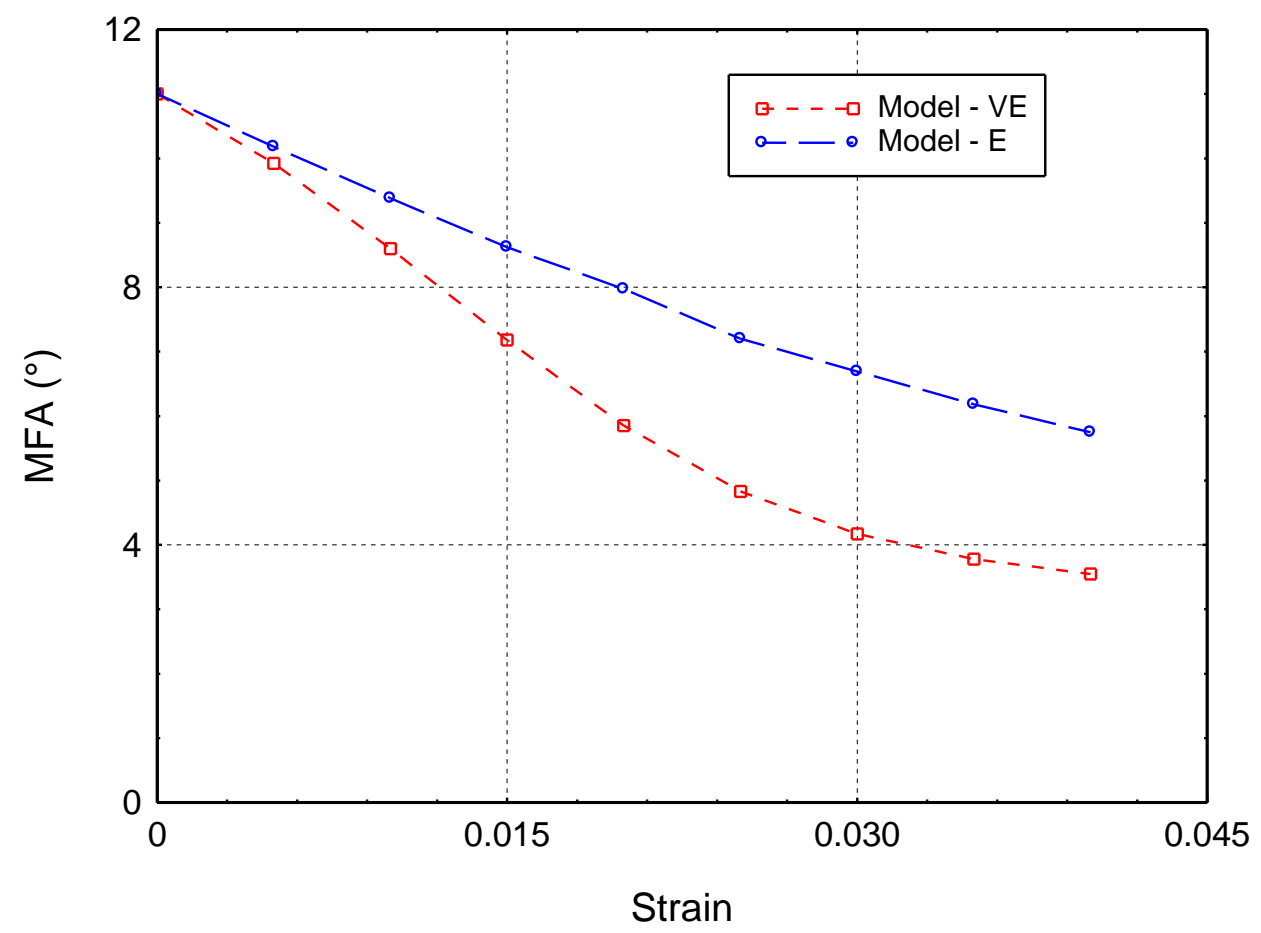


Figure 7. a. Computed stress-strain curves. b. Apparent tangent modulus - strain curves. The curves are computed for different strain rates from $5.10^{-5}$ to $5.10^{-3} \mathrm{~s}^{-1}$.
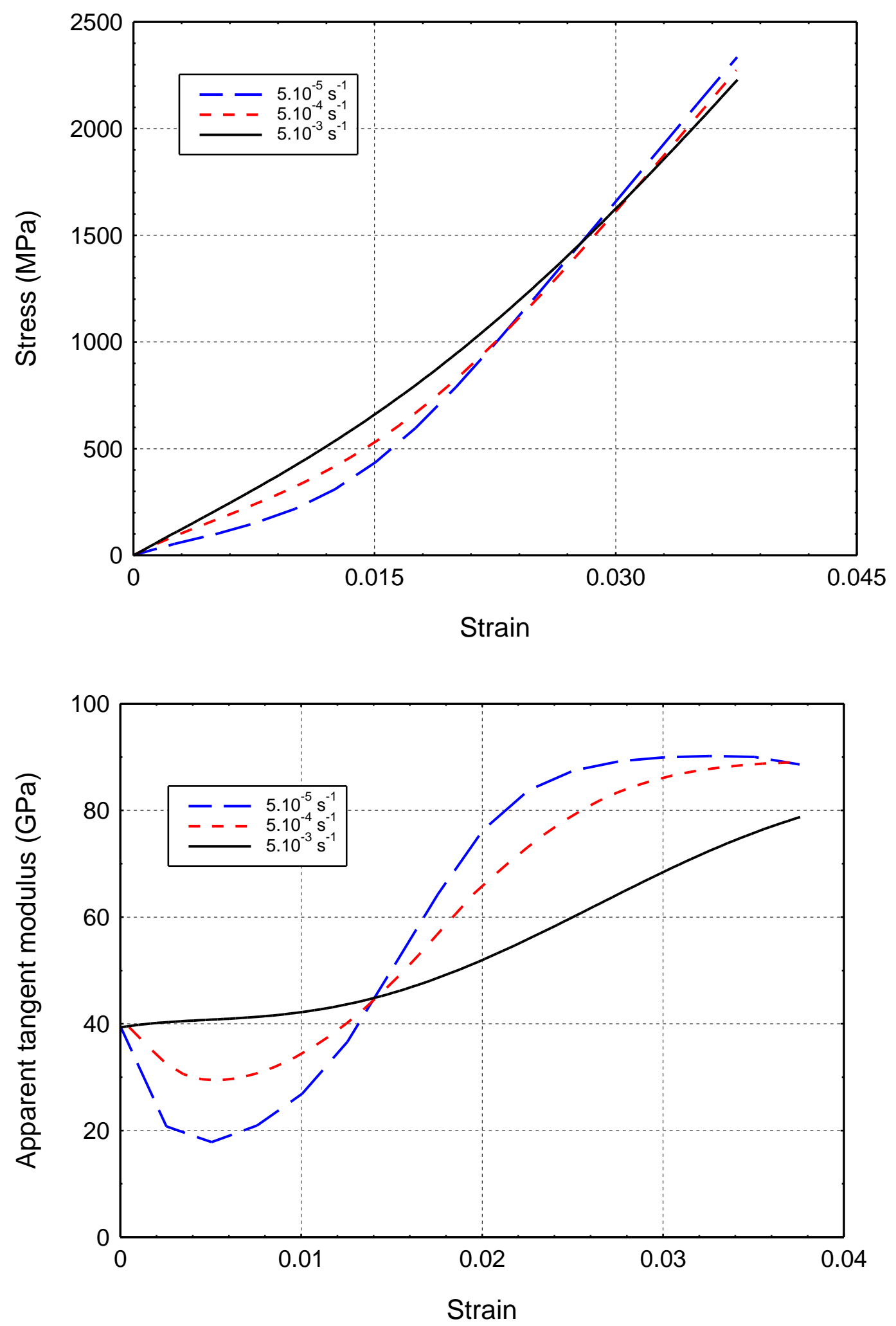
Figure 8: Computed MFA as a function of axial strain for two fibre areas: bulk fibre and dislocation area, using the VE constitutive law.

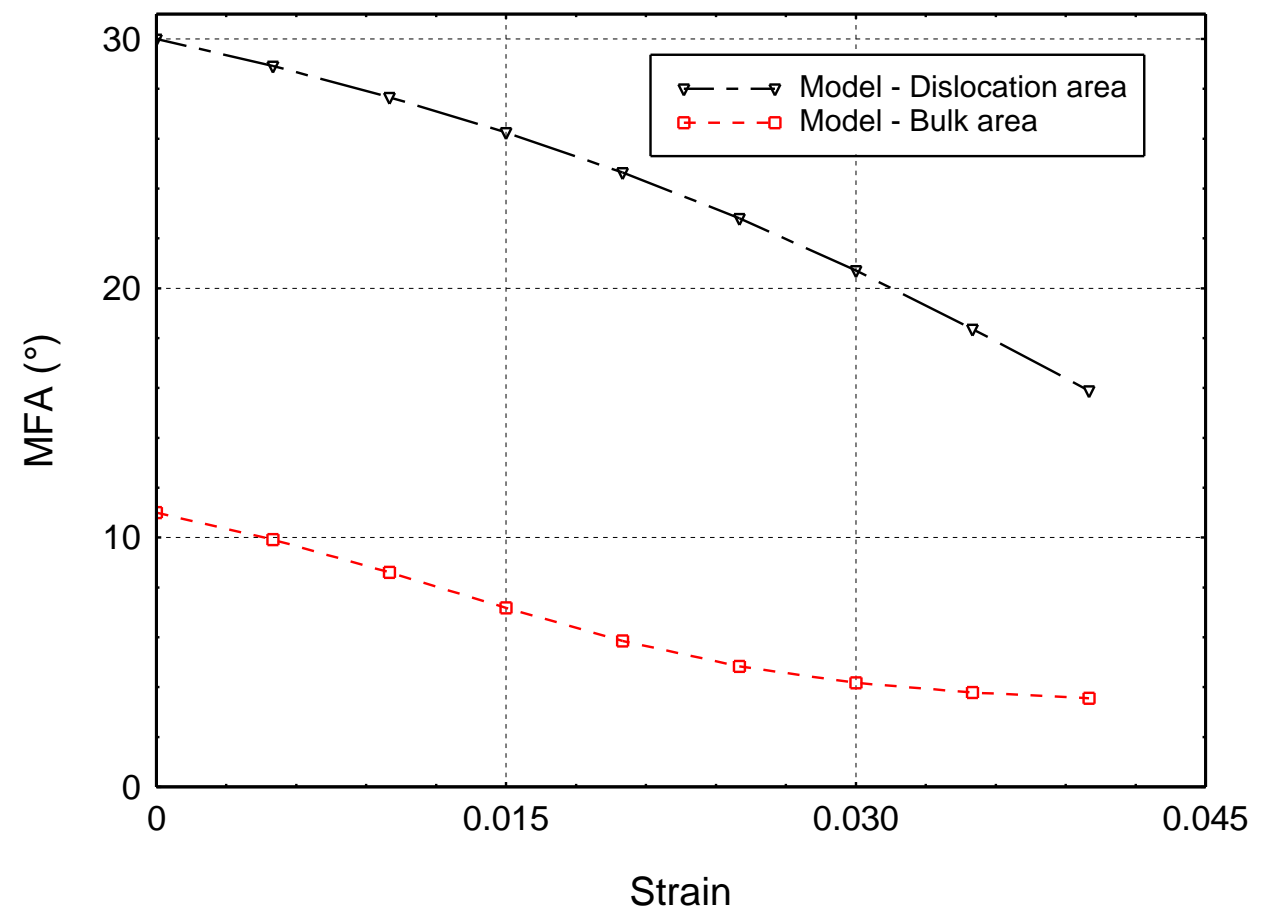


Figure 9: Computed axial stress as a function of axial position (a) and radial position (b), for a single fibre with a dislocation area (dislocation area: $25 \%$ of the fibre, $\mathrm{MFA}_{\text {bulk fibre }}: \mathbf{1 1}^{\circ}$, $\mathrm{MFA}_{\text {dislocation: }} \mathbf{3 0}^{\circ}$, based on the use of two different constitutive laws: $\mathrm{E}$ and VE/MFR/C.

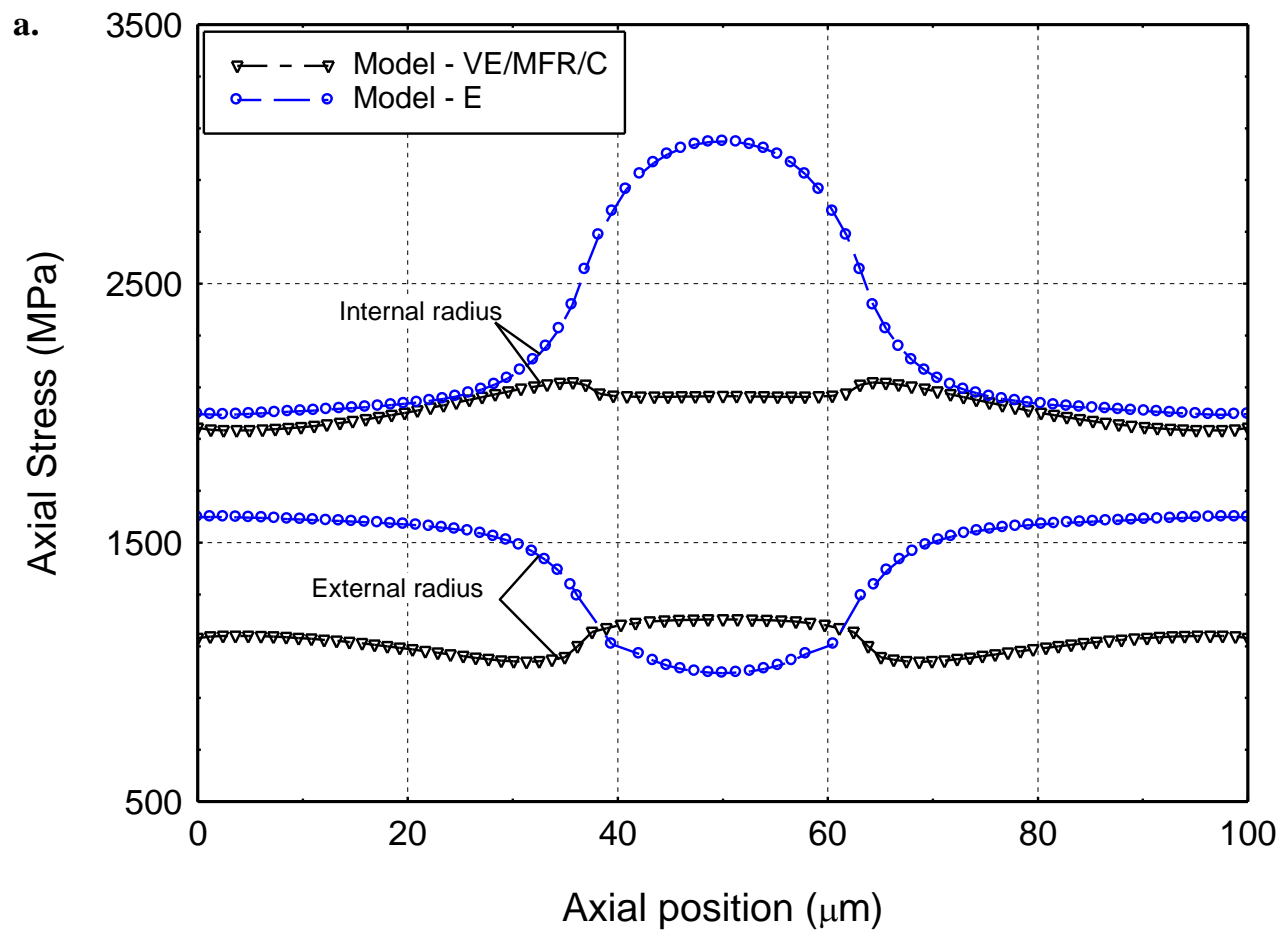

b.

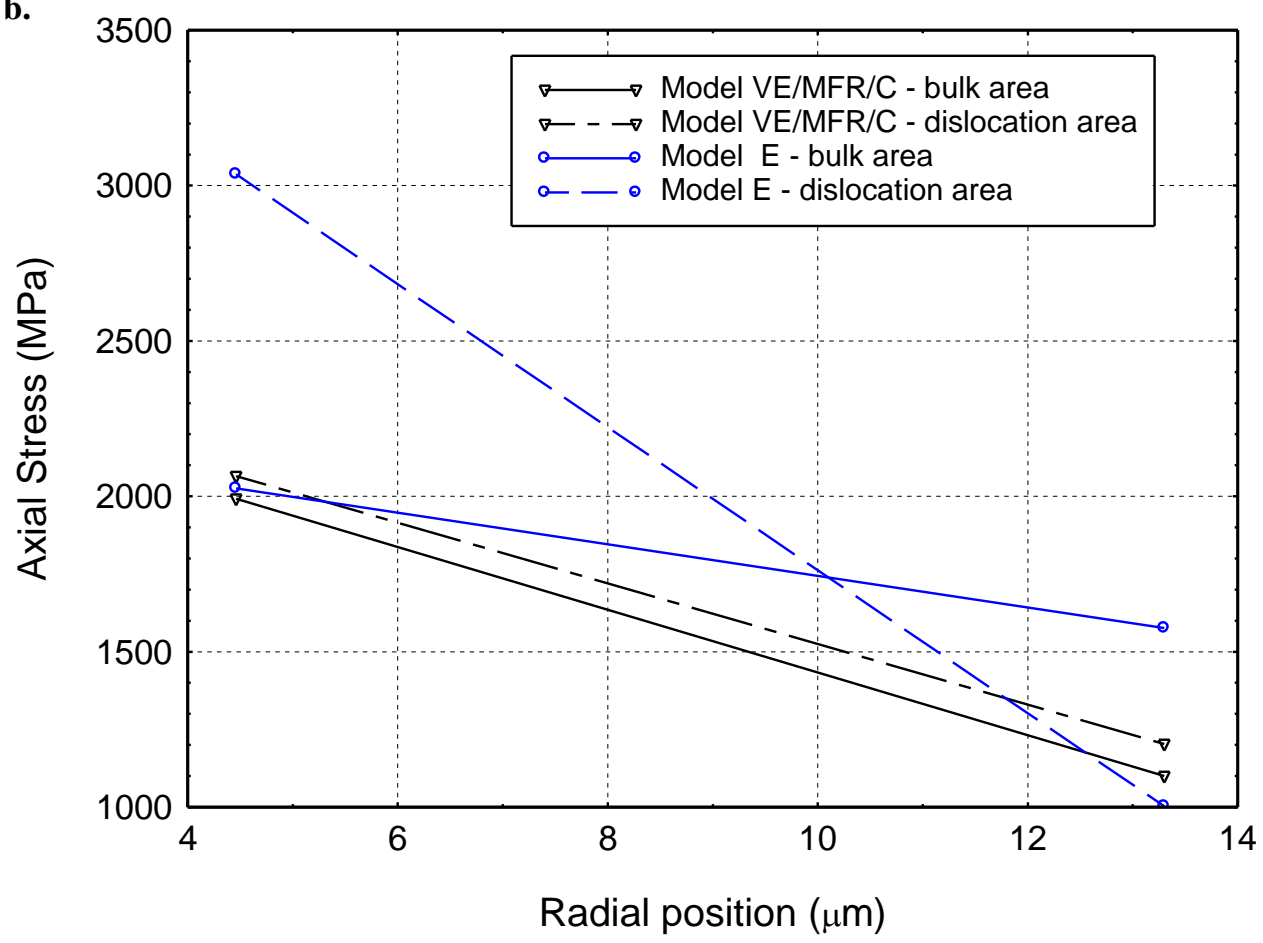


Figure 10: Computed tensile stress and strain fields for a fibre including a dislocation area (dislocation area: $25 \%$ of the fibre, $\mathrm{MFA}_{\text {bulk fibre }}: \mathbf{1 1}^{\circ}$, $\mathrm{MFA}_{\text {dislocation }}: 30^{\circ}$ ), assuming two different constitutive laws: $\mathrm{E}$ (a.) and VE/MFR/C (b.).

a.
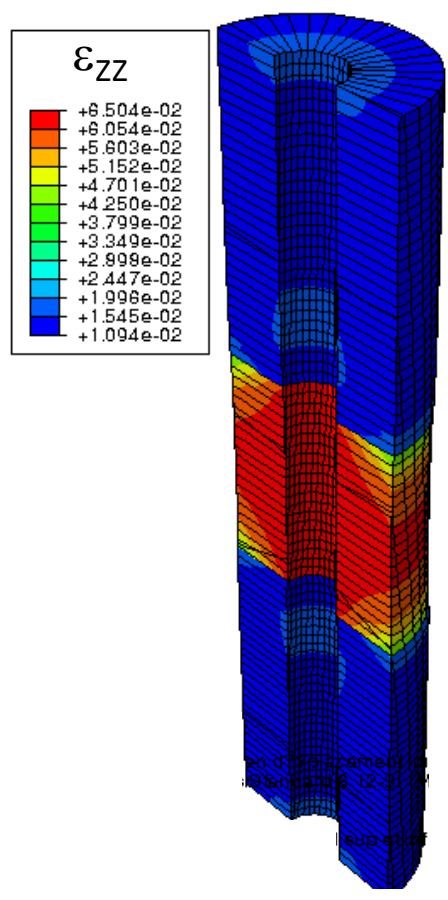

b.

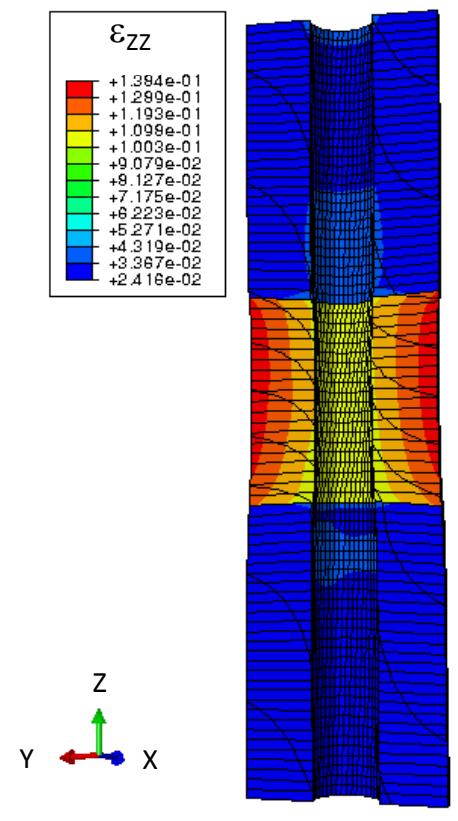

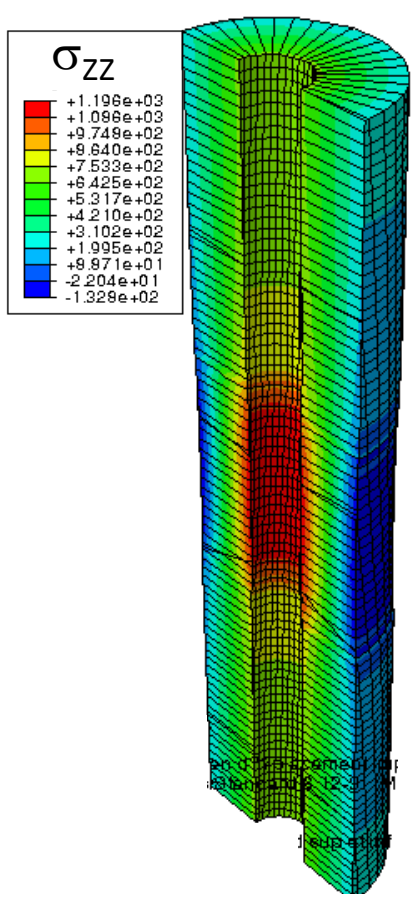
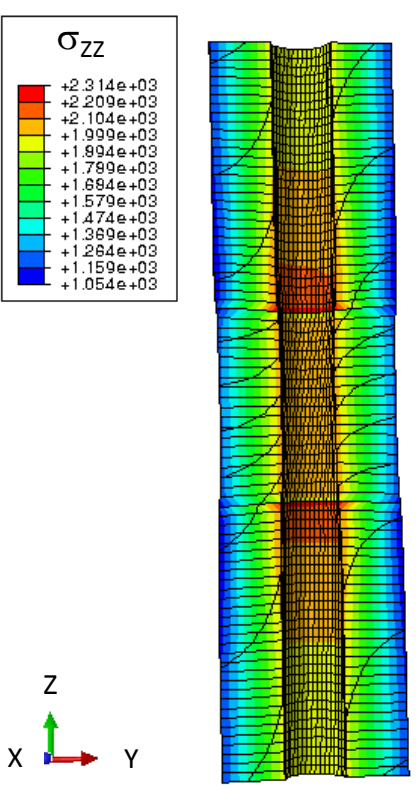
Table 1: Values of the model parameters used in the FE computations.

\begin{tabular}{|c|c|c|c|}
\hline \multicolumn{2}{|c|}{ Elastic parameters } & \multicolumn{2}{|c|}{ Viscoelastic parameters } \\
\hline $\mathrm{E}_{\mathrm{L}}$ & $75000 \mathrm{MPa}$ & $\beta_{\mathrm{LT}}$ & 12.25 \\
\hline $\mathrm{E}_{\mathrm{T}}$ & $11000 \mathrm{MPa}$ & $\boldsymbol{\beta}_{\mathbf{T}}$ & 1.5 \\
\hline$v_{\mathrm{LT}}$ & 0.153 & $\mathbf{z n}_{\mathbf{c}}$ & 2.45 \\
\hline $\mathrm{G}_{\mathrm{LT}}$ & $2520 \mathrm{MPa}$ & $\mathbf{z n}_{\mathbf{0}}$ & 1.9 \\
\hline$v_{\mathrm{TT}}$ & 0.2 & & \\
\hline
\end{tabular}

